\title{
Anabases
}

ANABASES Traditions et réceptions de l'Antiquité

5 | 2007

Varia

\section{Charles de Brosses entre Salluste et le patrimoine antique de Rome}

Philippe Foro

\section{OpenEdition}

1 Journals

Édition électronique

URL : http://journals.openedition.org/anabases/3163

DOI : 10.4000/anabases.3163

ISSN : 2256-9421

Éditeur

E.R.A.S.M.E.

Édition imprimée

Date de publication : 1 mars 2007

Pagination : 149-159

ISSN : 1774-4296

\section{Référence électronique}

Philippe Foro, «Charles de Brosses entre Salluste et le patrimoine antique de Rome », Anabases [En ligne], 5 | 2007, mis en ligne le 01 janvier 2012, consulté le 21 octobre 2019. URL : http:// journals.openedition.org/anabases/3163 ; DOI : 10.4000/anabases.3163

(c) Anabases 
Anabases 5 (2007), p. 149-159

\section{Charles de Brosses entre Salluste et le patrimoine antique de Rome}

PHILIPPE FORO

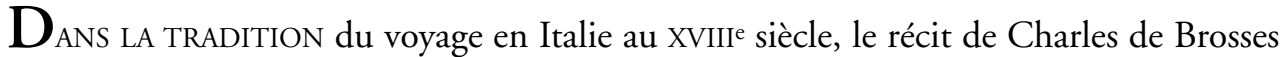
occupe une place de choix ${ }^{1}$. Dans son ouvrage L'Italie en 1818, Stendhal écrit : "Excepté de Brosses, les voyageurs ne se sont pas doutés des mœurs, des habitudes, des préjugés, des diverses manières de chercher le bonheur du peuple qu'ils traversaient, ils n'ont vu que les murs ${ }^{2}$. " Le lecteur contemporain bénéficie depuis peu d'une réédition des Lettres d'Italie du Président de Brosses ${ }^{3}$. Né à Dijon en 1709, à l'extrême fin du règne de Louis XIV, son existence traverse celui de Louis XV pour se terminer au début de celui de Louis XVI, en 1777. Après des études chez les Jésuites où il se lie d'amitié avec Buffon, il est reçu conseiller au Parlement de Bourgogne en 1730. Traducteur de La conjuration de Catilina de Salluste, il est admis comme correspondant de l'Académie des Inscriptions et Belles-Lettres en 1746. Sur le plan politique, il refuse de participer au Parlement issu de la réforme du Chancelier Maupeou en 1771. À l'apogée de sa carrière parlementaire, de Brosses devient Premier Président du Parlement de Bourgogne à la suite du rétablissement des anciens Parlements par Louis XVI en 1774.

Issu de la noblesse parlementaire, de Brosses apparait comme un esprit indépendant. Catholique mais facétieux, il n'hésite pas à ironiser, dans une lettre du 10 octobre 1739, sur la longue agonie de Clément XII : "Que dites-vous de la galanterie de notre

1 Sur le voyage en Italie, lire les textes rassemblés in Y. HERSANT, Italies. Anthologie des voyageurs français aux XVIII et XIXe siècles, Paris, collection Bouquins, Robert Laffont, 1996.

2 Cité par P. BRUnel dans sa préface de Stendhal, Rome, Naples et Florence, Paris, Folio classique, 1997 , p. 8.

3 Lettres d'Italie du Président de Brosses, Texte établi, présenté et annoté par F. D'AGAY, Le temps retrouvé, Paris, Mercure de France, 2005. Nos citations sont tirées de cette édition, ainsi que les renseignements biographiques sur Charles de Brosses. 
Saint-Père qui a la politesse de se laisser mourir pour nous faire voir un conclave 4 ? " Il sait également utiliser la hauteur de ton afin de faire comprendre à son interlocuteur son désaveu personnel. Ainsi, lorsqu'il doit prononcer une harangue à l'occasion de la visite du comte de Saulx-Tavanes, lieutenant-général du roi en Bourgogne et qui s'est heurté au Parlement sur des questions de préséance. Celui-ci est un discours d'accueil minimal. « Monsieur, le Roi, seul maitre des honneurs, ayant bien voulu vous accorder la plus grande distinction que vous puissiez recevoir en cette province, le Parlement toujours plein de respect et de soumission pour ses volontés, vient à l'occasion de votre retour exécuter l'ordre de Sa Majesté. » Une autre indéniable caractéristique de Charles de Brosses qui transparaît dans sa correspondance est sa solide culture classique. Dans une lettre en date du 2 novembre 1739, il cite des extraits de L'Éneide de Virgile, des Odes et Satires d'Horace. Dans celle envoyée à Buffon le 30 novembre, il décrit le Vésuve en s'appuyant sur Strabon et Pline le Jeune. Il est aussi, comme bien des hommes de l'élite intellectuelle et sociale du XVIII siècle, ce que l'on appelle un antiquaire. Il est souvent à la recherche du bel objet comme lors de sa visite à un prélat, futur Clément XIII. «Autre visite au cardinal Rezzonico, dans le palais Altemps ${ }^{5}$, non pas tant pour l'amour de lui, quoique honnête homme et civil, que pour l'amour de certains antiques, et surtout d'une célèbre Bacchanale en bas-relief ${ }^{6}$. " Dans une lettre à un de ses amis, M. de Quintin, il se montre capable d'établir un catalogue alphabétique, sans commentaire (et donc fastidieux), des principales peintures et sculptures se trouvant dans les églises, palais et villas de Rome ${ }^{7}$. Cependant l'homme n'est pas sans défaut. Ce merveilleux connaisseur de l'Italie qu'est Dominique Fernandez note sa "suffisance bornée " lorsqu'il traite les castrats italiens de « chapons ${ }^{8}$ ".

C'est le 30 mai 1739 que Charles de Brosses quitte Dijon pour entamer son périple italien. Celui-ci est l'occasion d'entretenir une correspondance avec quelques proches tels Buffon, Charles-Claude de Blancey, député aux États de Bourgogne ou Jean de Bouhier, Président à mortier du Parlement de Bourgogne. De ces nombreuses lettres, nous allons retenir trois aspects qui reviennent souvent et qui comptent parmi les intérêts majeurs de l'œuvre de Brosses. Tout d'abord sa quête d'une documentation sur Salluste, objectif officiel du voyage en Italie. Ensuite, la représentation que donne de Brosses de l'Antiquité romaine dont la grandeur et la beauté des monuments la place

$4 \quad$ Lettres d'Italie du Président de Brosses, op. cit., volume I, lettre XXVII, p. 395.

5 Situé entre la place Navone et le Tibre, le palais Altemps a été édifié sous le pontificat de Sixte IV dans les années 1480 mais profondément remanié par Martino Longhi à partir de 1568.

6 Lettres d'Italie du Président de Brosses, volume II, lettre XLI, p. 128-129.

$7 \quad$ Ibid., lettre LIII, p. 384-483.

8 D. Fernandez, Le voyage d'Italie, Paris, Perrin, 2004, p. 172. 
sur un piédestal à ses yeux ${ }^{9}$. Enfin, le témoignage qu'il nous offre du patrimoine de la Rome antique à la fin des années 1730 et que les travaux des XIXe et XXe siècles ont profondément modifié. Les lettres de Charles de Brosses forment un formidable arrêt sur image de la Rome du pontificat finissant de Clément XII (le pape meurt le 8 février 1740 alors que de Brosses est dans la Ville Éternelle).

\section{À la recherche des manuscrits de Salluste}

Le prétexte officiel du voyage en Italie est la quête de manuscrits de Salluste. Né en 86 avant J.-C., Caius Sallustius Crispus mène une vie publique au sein du parti césarien qui lui permet d'accéder aux postes de questeur en 55, de tribun de la plèbe en 52, de gouverneur de la province de l'Africa Nova en 46. Après le meurtre de César, Salluste se retire de la vie publique, profitant de sa villa et de ses jardins situés entre le Quirinal et le Pincio ${ }^{10}$, que son enrichissement, lors de son passage en Afrique, lui permet d'aménager. De 43 à 38, il rédige La conjuration de Catilina, La guerre de Jugurtha et les Histoires qui narrent l'histoire de Rome entre 78 et 67 et dont il ne reste que des fragments. Ayant l'intention de proposer une traduction de La conjuration de Catilina et une biographie de Salluste, Charles de Brosses signale régulièrement dans sa correspondance ses recherches et trouvailles. Il s'imprègne des lieux de l'histoire romaine décrite par Salluste telle la prison Mamertine, située sous l'église San Pietro in Carcere où fut emprisonné Jugurtha et les partisans de Catilina : "Jugez avec quelle avidité $j$ ’y suis descendu pour voir le spectre du roi de Numidie. Le lieu est encore, mot pour mot, tel que le décrit Salluste ${ }^{11}$. » De même, il dessine les traits de Marius dont la statue est aux musées Capitolins afin de les placer lors d'une description du dictateur prévue dans son Salluste.

D'autre part, de Brosses parcourt les bibliothèques à la recherche des manuscrits de l'historien romain. Dès avant de parvenir dans la Ville Éternelle, il se rend à la bibliothèque de l'université de Turin, fondée en 1405 par les ducs de Savoie, où « il y a assez de manuscrits, entre autres un très beau Lactance qu'on prétend être l'original sur lequel

9 Une exception cependant. La traversée de Vienne ne lui fournit pas l'occasion de souligner le patrimoine antique de la ville du Dauphiné (temple d'Auguste et de Livie, théâtre...). « Nous n'y trouvâmes rien de supportable que l'église Saint-Maurice, cathédrale bâtie dans un assez méchant goût gothique ", écrit-il dans une lettre en date du 6 juin 1739.

De Brosses se promène dans les jardins de la Villa Ludovisi situés à l'emplacement des jardins de Salluste : « Nous nous y rendons tous les soirs, à l'heure de la promenade ; jugez du plaisir ; ce sont les jardins de Salluste. Ceci vaut la peine d'en parler, et j'en ferai une mention honorable dans la vie de mon vieil ami que j'écris actuellement ". Lettres d'Italie du Président de Brosses, volume II, lettre XXXIX, p. 69.

11 Ibid., volume II, lettre XLI, p. 227. Pour la description chez Salluste, voir La conjuration de Catilina, LV. 
il a été imprimé ; j'en ai mis, selon ma coutume, quelques-uns de Salluste à part ${ }^{12}$ ». De même, à Milan, à la bibliothèque Ambrosienne, créée par le cardinal-archevêque Federico Borromeo, successeur de son cousin Charles Borromée en 1584, il retire "diverses collations de manuscrits de Salluste 13 ». À Florence, à la bibliothèque des Médicis dont Michel-Ange fut le premier architecte en 1524, il fait copier une vingtaine de manuscrits. Une fois à Rome, il va travailler à la bibliothèque de la Minerve, attenante de l'église des Dominicains, Santa Maria sopra Minerva: "Le vaisseau est grand, clair, commode, distribué à deux étages par une tribune, comme chez le Roi. Elle est publique, presque toujours remplie de gens qui travaillent. J'y ai trouvé d'excellents manuscrits de Salluste que l'on me collationne actuellement. On y est bien servi et de bonne grâce ${ }^{14}$. " Surtout il étudie à la bibliothèque Vaticane, créée par Sixte IV en 1475, dont il nous décrit le fonctionnement, l'atmosphère et les lieux. «La fameuse bibliothèque du Vatican n'est pas publique ; c'est la bibliothèque particulière du Pape, qui ne s'ouvre pas pour tout le monde ; mais, pour peu que l'on soit connu, on y est reçu fort poliment, et l'on $y$ va travailler cetains jours de la semaine dans une grande antisalle où les sous-bibliothécaires vous font apporter de la bibliothèque, les manuscrits dont vous avez besoin. " Concernant Salluste, il trouve "d'excellents manuscrits d'une antiquité marquée, un surtout appartenant à Fulvius Ursinus, et depuis à la reine de Suède ${ }^{15}$ ".

Pourquoi travailler sur Salluste ? Laissons de côté l'argument, malicieux et tendancieux, qui consiste à souligner la relative brièveté de l'œuvre de celui-ci. Plus fondamentalement, il faut sans doute prendre en compte l'influence de l'éducation reçue des Jésuites, héritiers en cela des Pères de l'Église - et saint Augustin le premier - pour qui " seuls Cicéron et Virgile le surpassent en considération " selon le constat établi par Ronald Syme, biographe de Salluste ${ }^{16}$. Dans le Ratio studiorum de 1599 qui organise la pédagogie des Pères, la lecture et l'étude de Salluste sont recommandées aux côtés de celles de César, Tite-Live, Florus et Eutrope. Pour les régents des collèges jésuites, l'étude de La conjuration de Catilina permet une réflexion sur la morale politique et l'exercice du pouvoir ${ }^{17}$. D'autre part, Salluste est un des auteurs régulièrement cités par les élites intellectuelles du Siècle des Lumières. Montesquieu le cite dans ses

12 Lettres d'Italie du Président de Brosses, volume I, lettre LVIII, p. 577.

13 Ibid., p. 564.

14 Lettres d'Italie du Président de Brosses, volume II, lettre XLI, p. 117.

15 Ibid., lettre XLVII, p. 256-257. La description de la Vaticane se trouve aux pages 255-261 et l'extrait cité se situe p. 255. La reine est Christine de Suède, convertie au catholicisme en 1654, installée à Rome, qui lègue sa bibliothèque à la Vaticane à sa mort, en 1689.

16 R. Syme, Salluste, traduction de Pierre Robin, Annales littéraires de l'université de Besançon, Paris, Les Belles Lettres, 1982, p. 240.

17 Sur les Jésuites et Salluste, voir F. DE DAINVILle, L'éducation chez les Jésuites (XVIeXVIII e siècles), Paris, 1978 ainsi que E. FLAMARION “ Fides an perfidia? Salluste et les régents jésuites dans la France du XVIII siècle " in Présence de Salluste, actes du colloque de Tours édités par R. Poignault, université de Tours, 1997, p. 143-155. 
Considérations sur les causes de la grandeur des Romains et de leur décadence (1734). Diderot l'utilise dans l'Encyclopédie et dans Les Principes de la politique du souverain. Voltaire, avec qui de Brosses correspond sur Salluste par une lettre du 2 janvier 1760, écrit une tragédie intitulée Catilina ou Rome sauvée. En choisissant de travailler sur Salluste, de Brosses ne fait donc pas preuve d'une véritable originalité. Les recherches entreprises sur Salluste par le parlementaire bourguignon aboutissent à une traduction de La conjuration de Catilina qui vaut à son auteur d'être désigné, en 1746, comme correspondant de l'Académie royale des Inscriptions et Belles-Lettres. Puis, en 1777, quelques semaines avant de mourir, Charles de Brosses publie, à Dijon, son Histoire de la République romaine dans le cours du VII siècle, regroupant près de sept cents fragments des oeuvres de Salluste, accompagnés par une biographie de celui-ci ${ }^{18}$.

\section{Splendeurs de la Rome antique}

Qu’y a-t-il de plus grand que ce que les Romains ont laissé ? Aux yeux de Charles de Brosses, les réalisations romaines sont les étalons qui permettent de juger les réalisations postérieures. Passé par Toulon, il écrit depuis Gênes, le 28 juin 1739 : «L'arsenal de Toulon ne vaut pas celui de Marseille, mais la corderie est bien au-dessus et vaut un ouvrage des Romains ${ }^{19}$. » De même à Vérone, le 25 juillet, devant l'amphithéâtre : «Je me confirme tous les jours dans l'idée qu'il n'y a eu que les Romains qui aient su faire des ouvrages publics ${ }^{20}$.» Ou encore à Naples, le 2 novembre, où il disserte sur les aqueducs : "C'est une chose surprenante que les ouvrages de ces Romains; on ne se lasse point d'admirer la grandeur de leurs entreprises qui est une preuve de celle de leur génie. Tous ces aqueducs sont composées d'une quantité prodigieuse d'arcades longues et étroites formées par des piliers et des voûtes de briques au-dessus desquels, comme sur une terrasse, court le canal qui va prendre les eaux à leur source pour les amener à leur destination 21. " Une promenade sur la via Appia l'amène à louer l'esprit public des anciens Romains : « [...] Un grand lambeau de la via Appia, long de plus de quarante milles est plus digne d'admiration que tout ce que l'on pourrait voir au monde, puisque le bien public en a été le motif ${ }^{22}$. " Cet amour des constructions romaines participe à sa répugnance envers l'art médiéval, sentiment assez commun à bien des hommes des Lumières. «Elle est noire, obscure, par-dessus tout cela gothique. Quelque magnifique

18 Il convient de souligner que cette œuvre de Salluste prend place dans le répertoire établi par A.D. LeEMAN, A systematical bibliography of Sallust, Leiden, Brill, 1952, p. 20. Par contre, Alfred Ernout, dans son introduction de l'édition des œuvres de Salluste (Paris, Les Belles Lettres, CUF, 1941), n'évoque pas le travail de de Brosses. Lettres d'Italie du Président de Brosses, volume I, lettre IV, p. 92.

Ibid., lettre XII, p. 181.

Ibid., lettre XXIX, p. 413.

Ibid., lettre XXX, p. 427. 
et d'un travail prodigieux que soit ce gothique, on redouble furieusement de mauvaise humeur contre lui quand on a vu les bâtiments des anciens Romains 23 ", écrit-il à propos de la cathédrale de Milan.

À Rome même, les monuments antiques, malgré un état de conservation souvent médiocre, surpassent bien des constructions moins anciennes : "Le peu qui reste de ceux-ci, défigurés comme ils sont, est encore autant au-dessus des modernes pour la simplicité et la grandeur que la République romaine était au-dessus de l'État de l'Église 24 . " Aussi peut-il être surpris par bien des aspects de la Rome de son temps. "Tout est de palais ou de cabanes ; un bâtiment superbe est entouré de cent mauvaises maisonnettes; quelques grandes rues principales, d'une longueur sans fin, alignées à merveille, presque toujours terminées par de beaux points de vue, aident heureusement à se retrouver au milieu d'une foule de culs-de-sac, de ruelles tortueuses ou de mauvais petits carrefours. Il n'y a rien de plus aisé que de savoir la ville en gros et rien de si difficile que de s'en démêler en détails ${ }^{25}$. " Mais ces lignes n'auraient-elles pas pu être écrites par Juvénal à propos de la Rome impériale ? Par contre, des sites romains attirent son attention. Le Corso, menant de la place du Peuple jusqu’à la place de Venise, dont de Brosses reconnaît la valeur (il venait d'être pavé à nouveau en 1736), est comparé à la via Flaminia dont il emprunte le parcours : "Toute belle qu'est aujourd'hui cette rue, combien est-elle déchue de son antique splendeur, si les plans et les descriptions qu'on nous donne de l'ancienne via Flaminia nous la représente telle qu'elle était, en effet, du temps des Romains! Que peut-on se figurer de plus magnifique et de plus frappant que cette double file de mausolées et de statues colossales qui régnait de côté et d'autre dans toute sa longueur, et de temps en temps, à droite et à gauche, des places, des colonnes, des obélisques 26 ? " Admirateur des fontaines de Rome, de Brosses rend hommage à la technique hydraulique des Romains. "Les aqueducs des anciens Romains, leurs égouts, leurs châteaux d'eau sont des ouvrages prodigieux. Il en a coûté, depuis deux ou trois siècles, des frais énormes pour en remettre en état une partie seulement, qui, avec quelques nouvelles adjonctions, a suffi pour fournir la ville d'une innombrable quantité de fontaines, grandes ou petites 27. " Au moment où de Brosses réside à Rome, les travaux de la fontaine de Trevi ont débuté

Lettres d'Italie du Président de Brosses, volume II, lettre LVIII, p. 564.

Ibid., lettre XXXVI, p. 11.

Cité in J.-Y. Boriaud, Histoire de Rome, Paris, Fayard, 2001, p. 292.

Lettres d'Italie du Président de Brosses, volume II, lettre XXVII, p. 18. Il est vrai que la via Flaminia prolongée par la vie Lata, était ornée de l'Ara Pacis, du cadran solaire d'Auguste, des arcs de Claude et de Dioclétien, des colonnes d'Antonin et de Marc-Aurèle. Mais, lucide, de Brosses ajoute : "Mais je soupçonne qu'on ne nous décrit que ce qu'il y avait de beau chez Messieurs les Anciens. De tous les vilains objets qui s'y pouvaient trouver, n'ayez pas peur qu'ils en disent mot".

Lettres d'Italie du Président de Brosses, volume II, lettre XXVII, p. 26. 
depuis sept ans (elle sera inaugurée en 1762), utilisant la construction d'Agrippa de l'aqua virgo desservant les thermes construits par le gendre d'Auguste, situés à proximité du Panthéon et des Saepta Julia. De plus, il a pu admirer la fontaine Paola, élevée entre 1610 et 1612, sous le pontificat de Paul V, ainsi que la fontaine du Triton, commandée par Urbain VIII au Bernin.

Par contre, les transformations, y compris la christianisation, de certains monuments de la Rome antique ne trouvent pas grâce auprès de Charles de Brosses. La sacralisation des thermes de Dioclétien en église des Chartreux ou Santa Maria degli Angeli où travailla Michel-Ange lui-même à l'extrême fin de sa vie, en 1563-1564, lui laisse des regrets. "Trois de ces salles sont antiques, faisant partie des restes des thermes de Dioclétien. Elles se sont trouvées toutes disposées de la sorte avec leurs colonnes, ce qui a fait naître la pensée d'en bâtir une quatrième pour terminer la croisée et en faire une église. C'est celle par où l'on entre. Michel-Ange en a été l'architecte mais elle est fort inférieure aux trois autres [...] ${ }^{28}$. " Ainsi pour le Panthéon, devenu l'église Santa Maria ad martyres, à la suite du don du bâtiment au pape Boniface IV par l'empereur byzantin Phocas en 608. "C'est un meurtre que d'avoir converti ce fameux temple en église. Il fallait laisser le Panthéon tel qu'il était, pour y placer dans les interstices des colonnes au-dedans et sous le portique au-dehors les plus belles statues antiques ${ }^{29}$. " Reproche identique envers l'utilisation du temple d'Hadrien comme Douane de Terre à la fin du XVII siècle, sous Innocent XII. "Quelle honte, au lieu de laisser ces exquises colonnes toutes sveltes et isolées, au lieu de réparer les bossages effacés des cannelures, d'avoir muré les intervalles par un infâme torchis, qui engage le vif des colonnes dans cette ignoble construction! Un assemblage aussi odieux est du dernier révoltant ${ }^{30}$. " Paradoxalement, il ne reproche pas au Bernin d'avoir utilisé les tuiles en bronze du Panthéon afin d'édifier le baldaquin au-dessus de la confession de Saint Pierre, dans la basilique Vaticane. Au contraire! « Mais le moyen de lui en vouloir du mal, quand on sait qu'il en a fait faire le superbe baldaquin de bronze du maître-autel de Saint Pierre, la plus belle pièce au monde en ce genre ${ }^{31}$."

\section{Témoignages sur les monuments antiques au XVIII siècle}

Pour l'étude de l'histoire des monuments de Rome, la correspondance de Charles de Brosses est un important témoignage qui nous donne une image arrêtée de ce qu'ils étaient en 1739-1740, avant les travaux de réhabilitations et les grandes transformations urbaines des XIXe et XXe siècles. Tout d'abord, notons que de Brosses peut commettre des erreurs comme lorsqu'il confond les morceaux de la statue géante de Constantin qui

Ibid., lettre XXXIX, p. 72.

Ibid., p. 60.

Ibid., p. 61.

Ibid., p. 60. 
ornait la basilique de Maxence, exposés dans la cour du palais des Conservateurs depuis 1487, avec des vestiges de la statue colossale de Néron ${ }^{32}$. De même, il confond, sur le Forum, le trois colonnes du temple de Castor et Pollux avec celles du temple de Jupiter Stator que la recherche récente place à l'emplacement de l'édifice appelé traditionnellement temple de Romulus, fils de l'empereur Maxence décédé en 307 33. Ou bien, il décrit la basilique de Maxence comme étant le temple de la Paix, édifié par Vespasien sur son forum ${ }^{34}$. Néanmoins, ses descriptions sont précieuses et nombreuses.

De Brosses fait état de monuments qui venaient de connaître une destruction récente ou qui ont disparu par la suite. Il évoque l'arc de Marc-Aurèle, près de l'église San Lorenzo in Lucina, proche du Corso : "depuis peu, il n'en reste plus rien "; il signale la fontaine de la Meta sudens, "tas de briques entre l'arc de Constantin et le Colisée ", qui fut définitivement rasée en 1936 par le gouvernement fasciste afin de faciliter les parades et revues organisées dans la zone du Colisée, de l'arc de Constantin et de la via dei trionfi (actuelle via San Gregorio), le temple du divin Claude, bâti par Agrippine et restauré sous Vespasien, nous précise de Brosses en citant Suétone " et dont il reste deux rangs de colonnes avec un portique au-devant 35 ». Le parlementaire bourguignon nous précise la situation de l'arc de Septime-Sévère, sur le Forum, avant qu'il ait été dégagé par les travaux que dirigera Carlo Fea, au début du XIXe siècle : "L'arc de Sévère est enterré presque jusqu'à la corniche de la grande porte du milieu, et à peu près jusqu'au sommet du cintre des deux petites portes des deux côtés, où l'on ne passe plus du tout ${ }^{36}$. " L'état de délabrement du Forum ne manque d'ailleurs pas de surprendre notre auteur. «Il est étonnant que, faisant autant de dépenses que l'on en fait ici pour rendre cette ville magnifique, on n'ait pas encore adopté un plan pour déblayer cette vaste place, lui donner forme, déterrer, rétablir, conserver les anciens monuments dont elle est remplie, et donner un bel aspect à ce lieu, qui contient tant de belles choses anciennes et modernes 37 . " Il est vrai que depuis les aménagements faits pour le passage de l'empereur Charles Quint, en 1546, afin qu'il traverse le Forum entre les arcs de Titus et de Septime-Sévère, rien n'avait été entrepris si ce n'est la plantation de deux rangées d'ormes au début du XVII siècle. Également instructive pour la connaissance des transformations de Rome est la description de la tombe de Bibulus et du Septizodium Severi. Le monument funéraire de l'édile Caius Poplicius Bibulus se situe au pied du Capitole, daté des premières années du Ier siècle avant J.-C., aujourd'hui isolé à proximité du monument à Victor Emmanuel II édifié entre 1885 et 1911 mais dont la construction a nécessité la destruction d'une zone d'habitation vue et décrite par de Brosses. La tombe «fait partie aujourd'hui d'une petite maison particulière dans une rue étroite qui monte au Capitole,

Ibid., lettre LII, p. 374.

Ibid., p. 375-376.

Ibid., lettre XLVI, p. 230-231.

Ibid., lettre LII, p. 370-373.

Ibid., lettre XLVI, p. 228.

37

Ibid., p. 230. 
appelée palais Mantica 38 ». Le Septizodium décorait l'angle sud-est du Palatin, en direction de la via Appia, formant un impressionnant nymphée construit sur plusieurs étages de colonnes à l'époque de Septime-Sévère. Presque intact jusqu'à la fin du XVIe siècle, le Septizodium est largement détruit sous le pontificat de Sixte V (1585-1590) afin de réaliser la chapelle Sixtine de Santa Maria Maggiore. Lors du séjour de Charles de Brosses, " on achève depuis plusieurs années d'en détruire les restes. C'était un édifice composé de sept portiques en terrasses, les uns sur les autres, et qui dominaient les plus hauts bâtiments de Rome 39 ".

Bien sûr, de Brosses porte un intérêt tout particulier aux grands édifices de la Rome antique. La description du Colisée est empreinte de révérence et de détails sur son utilisation quelques années avant que Benoît XIV, lors de l'Année Sainte 1750, ne sacralise l'amphithéâtre en interdisant toute utilisation comme carrière de pierres et lieux d'habitations par respect pour les martyrs qui auraient pu y perdre la vie (l'édification d'une église fut envisagée mais le projet ne vit jamais le jour sur les conseils du commissaire pontifical aux Antiquités, Ridolfino Venuti ${ }^{40}$ ). "En vérité, je crois qu'il est difficile de se trouver pour la première fois au milieu des augustes solitudes du Colisée et de l'Antoniane ${ }^{41}$, sans ressentir dans l'âme quelque petit saisissement, à la vue de la vieille majesté de leurs antiques masses révérées et abandonnées. Les galeries de l'enveloppe extérieure du Colisée servent encore néanmoins de refuge aux petits marchands, qui étaient sur des perches, fichées dans ces trous, d'où je vous ai dit que l'on avait tiré les tenons de bronze du sein des blocs de pierre. Il ne subsiste plus qu'un demi-cercle de cette enveloppe extérieure, à quatre prodigieux étages d'architecture en arcades et colonnes, le premier étage en partie enterré ${ }^{42}$. "Émotion identique devant ce qui était considéré comme la porte d'entrée de la demeure de Cicéron, en contre-bas du Palatin. «Voyez-vous, près de l'arc de Constantin, cette pauvre porte cochère ronde et basse ? Prosternez-vous, Quintin, c'est la porte de la feue maison de Cicéron. La place par où le maître de la république romaine rentrait chez lui, précédé de douze licteurs et suivi de deux mille chevaliers romains, n'est plus que le chétif atrium de quelques vignerons. Qu'est-ce que de nous ? Cela fait peur ${ }^{43}$."

De même, la promenade sur le Palatin donne à Charles de Brosses l'occasion d'écrire un beau passage sur la colline des Césars. "Presque toute cette vaste montagne où était le palais est occupée par la vigne Farnèse, assez négligée elle même. Elle est remplie de débris et de grandes pièces de marbre ; entre autres de fûts de grosses

Ibid., lettre LII, p. 382.

Ibid., p. 383.

Voir G. CiucCi (a cura di), Roma moderna, Storia di Roma dall'antichità a oggi, RomaBari, Laterza, 2002, p. 330-331.

Allusion aux thermes de Caracalla, le nom officiel du successeur de Septime-Sévère étant Marcus Aurelius Antoninus Bassianus.

Lettres d'Italie du Président de Brosses, p. 233.

Ibid., p. 235. 
colonnes de porphyre fendues et délitées en long par l'injure du temps. Tout cela est abandonné en plain air, quoiqu'on en puisse faire un bon usage, et le sera de plus en plus, la maison des Farnèse se trouvant éteinte. Cependant, le roi de Naples, héritier des Farnèse, et plus puissant qu'eux, avait beau jeu pour faire ici quelque chose de remarquable; mais c'est de quoi il ne se soucie guère 44 . [...] Les restes du palais d'Auguste 45 consistent en ces immenses voûtes dont je vous parlais, servant à soutenir le pied de l'édifice, et à mettre d'un même niveau sur ce terrain inégal, en une vaste salle impériale, jadis incrustée de marbre, ornée de colonnes et de pilastres corinthiens verts et jaune antique, dont les tores, les chapiteaux et la frise sont sculptés en bas-relief de la plus excellente beauté. Les tores sont en feuilles de chêne avec leurs glands, les bases et frises en figurines, trophées d'armes et d'arabesques d'un goût exquis ${ }^{46}$."

Par contre, de Brosses n’apprécie guère les catacombes. À son époque, celles-ci ont été redécouvertes ${ }^{47}$. Elles avaient connu une période d'oubli à partir du VIIIe siècle suite aux ravages provoqués par les Lombards en 756 , à quoi il convient d'ajouter le manque d'entretien des fidèles. Les travaux d'Antonio Bosio, à partir de 1593, participent à la renaissance des cimetières souterrains. Son ouvrage posthume de 1634 (Bosio meurt en 1629), dédié au pape Urbain VIII, est le premier travail qui peut être qualifié de tentative scientifique. D'autre part, en 1672, Clément X institue un cardinal-vicaire chargé de l'extraction des corps saints. Cependant, au sein même du monde catholique, des voix s'élèvent pour demander de la prudence dans la reconnaissance de corps de martyrs dans les ossements mis à jour. Ainsi, don Mabillon publie, en 1698, Lettre à Théophile, ouvrage mettant en garde les autorités romaines contre les risques d'abus. Pour sa part, de Brosses, outre le fait qu'il ne semble guère passionné par les catacombes, remet totalement en cause la tradition qui fait de ces lieux un refuge pour les chrétiens au moment des grandes persécutions. Le 24 novembre 1739, il écrit à son ami Neuilly : "D’une extrémité à l'autre, je vous précipite aux catacombes ; cela vous épargnera la peine de voir celles de Rome car ce ne sont pas des objets qui soient curieux deux fois ; moi qui vous parle, j’ai pourtant eu la sottise de voir encore celles de Sainte-Agnès; mais que mon exemple vous rende sage! Ce sont de longs corridors souterrains creusés dans des

44 La colline du Palatin a été achetée par Alessandro Farnèse en 1535 où il fit édifier le casino portant le nom de la famille. De 1722 à 1729, Francesco Farnese, duc de Parme entreprend des fouilles, sous la direction de Francesco Bianchini, afin de récupérer des objets d'art qui pouvaient être enfouis. En 1731, à la mort d'Antonio Farnese, décédé sans héritier, le duché de Parme et la possession du Palatin passent aux Bourbons espagnols en la personne de Don Carlos, fils de Philippe V d'Espagne et d'Élizabeth Farnese. Don Carlos devint roi d'Espagne en 1759 sous le nom de Charles III, après avoir été roi de Naples. En l'occurrence, plus que du palais d'Auguste, il s'agit du palais édifié à l'époque de Domitien, sous la conduite de l'architecte Rabirius.

Lettres d'Italie du Président de Brosses, lettre XLVIII, p. 274-275.

Que l'on nous permette de renvoyer à Ph. FORO, "La mémoire des catacombes de Rome ", Bulletin de littérature ecclésiastique, Institut catholique de Toulouse, CII, janviermars 2001, p. 25-38. 
carrières de pierre. D'un côté et de l'autre, la pierre est taillée en niche, comme une bibliothèque. On peut assurer avec certitude que ceci n'a jamais été fait que pour servir de cimetière, soit depuis qu'on eut quitté l'usage de brûler les corps ; soit peut-être même avant que cet usage ne fût introduit, du moins on le pourrait penser des catacombes de Rome. On logeait un ou plusieurs cadavres dans chaque niche, après quoi on la murait, selon les apparences, pour prévenir l'infection. C'est une folie ridicule que de dire qu'elles aient été creusées par les premiers chrétiens pour s'y loger et célébrer les saints mystères, à couvert de la persécution. Le joli logement, s'il vous plaît, que de pareilles galeries sans air et sans lumière 48 ! " Ce sont les écrivains du XIXe siècle qui revinrent sur les catacombes comme lieu de refuge au cours des persécutions, à l'image de Chateaubriand dans son roman de 1809, Les martyrs de Dioclétien, le cardinal Wiseman qui publia, en 1854, Fabiola ou l'Église des catacombes, le romancier polonais Henryk Sienkiewicz, auteur de Quo Vadis en 1896.

Charles de Brosses quitta Rome le 28 février 1740, assez tard pour avoir été présent au moment de la mort de Clément XII, mais trop pour avoir pu assister à l'élection de Benoît XIV. Au même titre que les dessins du Piranèse, les gravures de Pier Leone Ghezzi et Paolo Posi, les tableaux de Giovanni Paolo Panini, les lettres de Charles de Brosses sont un témoignage important sur la Rome du milieu du XVIII siècle comme aurait pu l'être le carnet, malheureusement perdu, consacré à son séjour romain, de l'architecte Pierre-Louis Moreau ${ }^{49}$. Il est également un des derniers exemples de ces aristocrates qui, sous l'Ancien Régime, participaient au Grand Tour dont l'Italie était une des étapes indispensables. Il en eut les préventions (son rejet du gothique est significatif) et les admirations, en homme de son temps. Son amour de Rome ne peut cependant pas l'empêcher d'observer combien Paris lui semble porteuse d'avenir au regard de ce qui apparaît comme la majestueuse immobilité de la Ville Éternelle. « [...] Elle est, quant au matériel, non seulement la plus belle ville du monde, mais hors de comparaison avec toute autre, même avec Paris, qui, d'autre côté, l'emporte infiniment pour tout ce qui se remue ${ }^{50}$. "Quelques dizaines d'années plus tard, ce ne fut pas la noblesse française qui fit le voyage de Rome mais les armées révolutionnaires et impériales.

\begin{tabular}{l} 
Philippe FORO \\
\hline Université de Toulouse II-Le Mirail \\
UFR d'Histoire, Arts et Archéologie \\
5, allées Antonio Machado \\
F-31058 Toulouse cedex 9 \\
philippe.foro@wanadoo.fr
\end{tabular}

48 Lettres d'Italie du Président de Brosses, volume I, lettre XXXI, p. 436-437.

49 Sur ce sujet, se reporter à S. DESCAT, Le voyage d'Italie de Pierre-Louis Moreau : journal intime d'un architecte des Lumières (1754-1757), Bordeaux, Presses universitaires de Bordeaux, 2004.

Lettres d'Italie du Président de Brosses, volume I, lettre XXXVI, p. 11. 